\title{
Expectativas de especialización profesional en estudiantes de odontología. Revisión de literatura
}

\author{
Expectations of professional specialization in dental students. Literature review
}

Matías Navarro-Mora ${ }^{1, a}$, Ricardo Cartes-Velásquez ${ }^{1, b, c, d, e}$

\section{RESUMEN}

El desarrollo profesional continuo se ha convertido en un objetivo común a todas las profesiones y la odontología no es una excepción, siendo las especialidades odontológicas las que representan el camino más importante para este desarrollo. La elección de la especialidad tiene fuertes implicancias no solo para el profesional o las comunidades educativas y científicas, sino también para la salud pública de los países. Por este motivo, es relevante conocer que elementos intervienen en el proceso de elección de la especialidad. El objetivo de este artículo es hacer una revisión de los factores y evolución de las preferencias de especialización entre los estudiantes de odontología, reportados en la literatura internacional reciente. Existen diversos motivos que influencian la decisión de especializarse y la elección de la especialidad, entre ellos se encuentran: estabilidad financiera y retribución económica, realización e intereses personales, reconocimiento social o "status", experiencia profesional, altruismo y carga de deuda de los estudiantes. Sin embargo, el peso de cada uno de estos factores es variable entre los distintos países e incluso al interior de estos. En América Latina con excepción de Brasil, la investigación en esta área ha sido escasa. Esto dificulta la generación de políticas para la formación de recursos humanos especializados en odontología que satisfagan las necesidades de la población. Por tanto, es necesario desarrollar investigación que permita comprender de mejor manera la elección de la especialidad de los actuales y futuros dentistas en la realidad de cada país o región.

PALABRAS CLAVE: Estudiantes de odontología, especialización, internado y residencia, educación en odontología. (DeCS, BIREME)

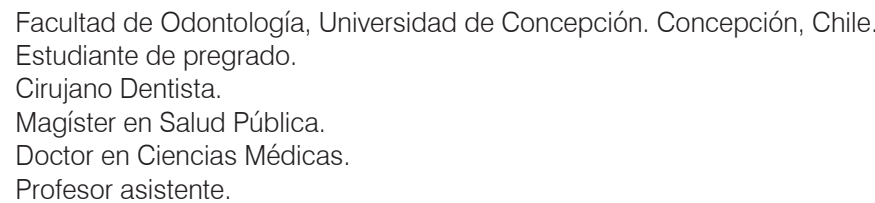




\section{SUMMARY}

Continuing professional development has become a common aim to all professions, and Dentistry is not the exception, being the dental specialties the most important way for this development. The choice of a specialty has strong implications not only for the professional or the educational and scientific communities, but also to the public health of the countries. For this reason, it is important to know which elements are involved in the process of choosing specialty. The aim of this article is to review the factors and evolution of preferences for specialization among dental students, reported in the recent international literature. There are several reasons that influence the decision to specialize and choice of a specialty, among them are: Financial stability and economic retribution, Development and personal interests, Social recognition or "status", Work Experience, Altruism and Student debt load. However, the weight of each of these factors varies between countries and even within these. In Latin America excepting Brazil, research in this area has been limited. This hinders the development of policies for the training of specialized human resources in Dentistry that meet the needs of the population. It is therefore necessary to develop research to better understand the choice of specialty of the current and future dentists in the conditions of each country or region.

\section{KEYWORDS: Students, dental; specialization; internship and residency; education, dental. (MeSH, NLM)}

\section{INTRODUCCIÓN}

El desarrollo profesional continuo se ha convertido en un objetivo común a todas las profesiones y la odontología no es una excepción. Las especialidades odontológicas representan el camino más importante para este desarrollo, asegurando el continuo crecimiento profesional, así como la adquisición de nuevas competencias entre los odontólogos (1).

Se ha comprobado que los estudiantes de odontología de nuevo ingreso ya tienen internalizada la idea: ser un especialista de la odontología da más prestigio que no serlo, sobre todo en determinadas áreas (2). Esta idea tiene fuertes implicancias no solo para el profesional o las comunidades educativas y científicas, sino también para la salud pública de los países. Por este motivo, es relevante conocer qué elementos intervienen en el proceso de elección de la especialidad.

El objetivo de este artículo es hacer una revisión de los factores y evolución de las preferencias de especialización entre los estudiantes de odontología, reportados en la literatura internacional reciente.

\section{REVISIÓN DE LITERATURA}

La especialización es considerada un avance y mejoramiento del profesional, en muchos aspectos es vista como la estabilidad financiera y el cumplimiento de expectativas o intereses personales. Por ello, resulta evidente que estas se transformen en motivaciones para tomar la decisión de especializarse y qué área elegir (3).

En general los estudiantes de las carreras de la salud, durante su formación, se ven expuestos a una gran variedad de opciones de especialización, teniendo un gran impacto las experiencias durante sus años de estudio; sin embargo, los factores que llevan a los estudiantes a elegir una u otra especialidad, o no especializarse son múltiples y complejos (4).

La decisión de elegir una especialidad se relaciona en términos generales con factores de diversa índole como: costo de la especialidad, el disfrute de proporcionar ese tipo de atención especializada, la exposición previa en pregrado, la influencia de la facultad, el salario futuro como especialista, la duración y ubicación del programa, tipos de pacientes que demandan atención en esa especialidad, entre otros (5).

Del mismo modo, se han reportado otros factores que hacen más interesante o atractiva la especialidad que van más allá de la retribución material, tales como: la complejidad, el desafío de la planificación del tratamiento y el desarrollo del mismo, la capacidad para dirigir los casos multidisciplinarios, la posesión de habilidades/talentos adaptados a la especialidad, el disfrute del trabajo clínico y el contenido intelectual de la especialidad (6). 
En el caso específico de la odontología, la Asociación Dental Americana (ADA) reconoce a nueve especialidades dentales: salud pública dental, endodoncia, patología oral, radiología oral y maxilofacial, cirugía bucal y maxilofacial, ortodoncia, odontopediatría, periodoncia y prostodoncia (7).

En una muestra de estudiantes hindúes se observó que la cirugía bucal fue la especialidad odontológica más buscada (8). En medicina existe concordancia con lo que ocurre en odontología, donde la cirugía es también la especialidad más elegida por los estudiantes. Así lo señala el estudio realizado en Arabia Saudita, donde esta especialidad tiene mayor preferencia, por encima de otras especialidades como medicina interna (4).

En los Emiratos Árabes se comprueba que la cirugía bucal y maxilofacial está dentro de las especialidades más perseguidas o preferidas; sin embargo, es finalmente la ortodoncia la más elegida por los estudiantes respecto a las otras especialidades. En relación a ortodoncia y cirugía, los estudios señalan que ambas están asociadas con mayores ingresos. Por lo tanto, proporcionarían estabilidad financiera y "estatus social", beneficios que aumentaría el atractivo para los estudiantes (3).

Respecto a la elección de la ortodoncia como especialidad, se ha visto disminuir con el tiempo, mientras que esta opción ha aumentado considerablemente en los últimos años para la implantología y estética (odontología cosmética). Sin embargo, existen importantes variaciones en esta elección (4).

Un estudio realizado en la India (8) encontró preferencias mayoritarias por la odontología conservadora, endodoncia, ortodoncia, cirugía bucal y maxilofacial, odontopediatría y periodoncia. Mientras que en Nigeria (9) las preferencias de los estudiantes fueron hacia las especialidades tradicionales (ortodoncia, cirugía bucal y maxilo facial). La radiología, anestesia dental, salud pública odontológica y medicina bucal no fueron consideradas en absoluto. Esta falta de interés en algunas especialidades ocasiona problemas a corto plazo, ya que el funcionamiento de un sistema de atención odontológico integral requiere de especialistas en todas las áreas (9). Y la situación se agrava cuando se consulta a decanos y académicos, quienes señalan a la prostodoncia y ortodoncia como las principales especialidades que debe tener una facultad; en claro desmedro por la salud pública y comunitaria (10).

Profesionales estadounidenses que cursaban la especialidad de cirugía bucal y maxilofacial indicaron que estaban satisfechos con la elección realizada y con las capacidades que venían adquiriendo, debido a que les ofrecía una gran mejora en su desempeño profesional (11). Es importante señalar que la especialización en cirugía bucal y maxilofacial no es homogénea a nivel internacional, ya que en algunos países es necesario un proceso de doble titulación; es decir, es necesario primero ser médico y luego odontólogo (12).

En Estados Unidos el mercado de trabajo para los especialistas de áreas específicas mejoró en gran parte debido a: la mayor prevalencia de enfermedades que requerían atención especializada, al uso de tecnología médica más avanzada y a la disminución de la satisfacción profesional de los médicos de atención primaria o familiar (13); esto provocó un aumento desproporcionado de especialidades como: cirugía general, obstetricia, ginecología y medicina de emergencia respecto a la tasa global de especialidades (14).

Del mismo modo que los estudiantes de medicina, los estudiantes de odontología no perciben a la salud comunitaria o familiar odontológica como una especialidad, debido a que es considerada como un área no clínica, menos prioritaria y atractiva como opción de especialización, a pesar que constituye la base de los servicios de cuidado de la salud dental y tiene la llave para el desarrollo de la conciencia sobre los aspectos sociales de la profesión y la responsabilidad para con la comunidad (9).

Se ha descrito que las expectativas de especialización se ven afectadas directamente por el sexo de los estudiantes. Las mujeres siguen eligiendo en una menor proporción las especialidades quirúrgicas, privilegiando especialidades que permitan un estilo de vida más flexible (15). Del mismo modo se ha reportado que la visión de la profesión, las expectativas del futuro financiero y la seguridad laboral se ven afectadas por el sexo de los estudiantes. Un estudio realizado en Estados 
Unidos, señala que los estudiantes varones del último año de pregrado tienen una perspectiva más positiva sobre la profesión, comparado con las mujeres. Se hizo evidente que los primeros generalmente tienen una actitud positiva hacia el "ser un dentista", producto del estatus social implícito, pero perciben a la profesión dental como estresante y exigente (17). Este estudio señala que existen algunos hechos innegables en la práctica clínica que son producto de las diferencias entre hombres y mujeres. Por una parte, la menor proporción de mujeres en el campo de la especialidad quirúrgica, y además que son los especialistas hombres quienes tienen mayor cantidad de pacientes. Sin embargo, con respecto al tipo y calidad de tratamiento, éste es evaluado como igual en ambos profesionales (17).

Dentro de las expectativas de especialización de los estudiantes de odontología, surge como una posibilidad deseable la idea de realizar estudios de posgrado o especialidad en el extranjero, debido a los beneficios académicos, sociales y económicos que conlleva $(16,18,19)$. En el caso de la India, fueron los estudiantes de universidades privadas quienes mostraron un mayor interés en realizar estudios fuera de su país (16). Garla (18), señala que el $62.17 \%$ de los estudiantes encuestados, tuvieron interés en ir al extranjero para realizar sus estudios de posgrado y especialidad, los países de mayor preferencia fueron: EE.UU, Australia, Reino Unido y Nueva Zelanda. En China, los estudiantes que querían especializarse y trabajar en el extranjero, lo hacían por tres razones principales: dinero, medio ambiente y búsqueda de un mejor sistema de atención médica (19).

\section{Motivaciones para especializarse}

Para comprender mejor estas motivaciones es necesario considerar el alto porcentaje de estudiantes que desean esta opción. Según Rashid y col. (3) a largo plazo un $92 \%$ de estudiantes de odontología plantea como su principal intención de carrera es especializarse (3). Otro estudio realizado en Brasil, evidenció que el $80.2 \%$ los estudiantes del último año de pregrado, tienen la intención de trabajar como especialista, mientras que el $11 \%$ están destinados a trabajar como dentista general y el $8.9 \%$ tienen la intención de trabajar en un entorno académico (20). Las estadísticas de la Escuela de Odontología de Harvard muestran que el $87.6 \%$ de graduados continúan su formación en los programas postdoctorales, el $72,9 \%$ en la especialización y el $14.7 \%$ en la formación odontológica avanzada (21).

Según Dhima y col. (5) existen cuatro factores generales que influirían en la decisión de especializarse y la elección de la especialidad, estos son: el salario, la calidad de vida personal, la calidad de vida del paciente y el impacto general sobre la profesión. Sin embargo, la literatura muestra una amplia diversidad de factores que intervienen en esta decisión, siendo posible identificar al menos seis. Estos son:

\section{Estabilidad financiera y retribución económica}

La mayoría de estudiantes considera la razón $\mathrm{f}$ inanciera como la influencia más importante en la toma de decisiones sobre la especialización a corto plazo, así como también que ayuda a conseguir un mejor estándar de vida (2). Otro factor muy valorado en la odontología es la "seguridad laboral", la obtención de una especialidad contribuye a esta idea de seguridad (21). La evolución que ha tenido la carrera conduce a los estudiantes a elegir una especialidad fijándose en las posibilidades del mercado en que puedan obtener un mayor ingreso (22).

Aun cuando la odontología representa un vehículo para el logro de metas personales, como una buena oportunidad de trabajo en el extranjero, independencia financiera y prestigio (7), la idea de futuro laboral de muchos odontólogos radica en la oportunidad de combinar la práctica dental privada y pública como una manera de lograr ingresos y la seguridad laboral (20).

Respecto a las posibilidades de ingreso, los estudiantes brasileros tienen una expectativa financiera realista de la profesión y no esperan un nivel de ingreso tan alto en el futuro, como sí lo fue hace pocas décadas atrás; esto se explica por el notable aumento de escuelas dentales en ese país. A la vez creen que especializarse les permitirá adquirir mayores y más complejas competencias en la atención clínica, lo cual traería una retribución económica (19).

En cuanto a las diferencias por genero, son los estudiantes varones quienes valoran mayormente 
el autoempleo y la retribución económica, mientras que las mujeres valoran más los motivos orientados a las personas como: el contacto con las personas, altruismo y la retribución personal (7). Otro estudio que avala esta afirmación, es el realizado por Lambert y Holmboe (23) donde se ha demostrado que las mujeres tienden a elegir especialidades en base a la realización personal y experiencias positivas, dejando en segundo lugar la retribución económica, mientras que los hombres dan mayor énfasis a las ventajas financieras y las habilidades de destreza manual.

\section{Realización e intereses personales}

Según Zarchy y col. (7), la elección de la odontología como carrera tiene una fuerte influencia de los padres, pero luego las decisiones son más bien personales y las influencias familiares pasan a un segundo plano. Gallagher y col. (24) encontraron que el "asesoramiento profesional" y la opinión de "amigos" y la "familia" fueron menos reconocidos como influencias en la decisión de especializarse, reforzando el concepto que es una elección personal (24).

Por otra parte, en un estudio realizado en Arabia Saudita, se encontró que el $62 \%$ de los estudiantes del último año de pregrado reportaron que su elección de su especialidad se vio influenciada por la obtención de buenas notas en una determinada materia, los intereses que presentaban por un área de la odontología y el tipo de profesional que les gustaría ser (4).

Las respuestas de los estudiantes frente a sus motivaciones se relacionan principalmente con sus intereses personales y aspiraciones, con puestos laborales de interés, calidad de vida y la flexibilidad, junto con la naturaleza de la ocupación y de la gama de oportunidades de la carrera (25).

\section{Reconocimiento social o "status"}

Históricamente el profesional de odontología tiene un demostrado prestigio y estabilidad social, situación que ha ido variando en las últimas décadas producto de la saturación profesional (26).
En Turquía, una menor -aunque no despreciableproporción de estudiantes considera que el estatus social y la reputación que les otorga la profesión odontológica es importante para poder cumplir con aspiraciones personales; además, el hecho de especializarse genera mayor respeto $y$ aceptación dentro de la profesión (27). Por otra parte, en Jordania, el prestigio y ayudar a las personas, fueron los factores motivadores más importantes en la elección de la carrera (3).

En India la admisión a medicina y odontología es altamente competitiva y requiere un alto resultado en la prueba de admisión. Los estudiantes de estas carreras suelen provenir en su gran mayoría de los estratos sociales más altos; por lo tanto, eligen carreras que les permitan mantener ese nivel social (28). En Chile ocurre una situación similar, los estudiantes de menor nivel socioeconómico se encuentran en menor número en la carrera de odontología (29). Esta situación de selectividad socioeconómica del pregrado tendría un impacto en la elección posterior de la especialidad, ya que se intentaría mantener o mejorar el estándar de vida previo (2).

\section{Experiencia profesional}

Las especialidades dentales requieren un alto nivel de formación y talento, por ello quienes hayan conocido y practicado alguna área de la odontología tendrían una tendencia por especializarse en esa área (30). Esta afirmación es avalada por Dastjerdi $\mathrm{y}$ col. (31), quienes sostienen que existe una relación entre la elección de una especialidad y la "experiencia en el trabajo"; es decir, la posibilidad que tendrían los estudiantes y odontólogos de relacionarse con las diferentes especialidades de la profesión durante su etapa de pregrado.

Fouad (30) sostiene que al elegir una especialidad influyen algunos factores, tales como: habilidades personales con las que cuenta el profesional y el interés por el área, que se complementa con la práctica clínica que adquiere el odontólogo. Esto llevaría a una mayor motivación producto de un estado de seguridad y compromiso con la especialidad elegida. 


\section{Altruismo}

En países como Finlandia, Australia y Dinamarca, los estudiantes señalaron razones altruistas, como son: las necesidades de la sociedad, el impacto de la profesión en la comunidad y la mejoría de salud de la población (22). Mehmood y col. (4) señalan que la mayoría de los estudiantes encuestados $(87 \%)$ consideraba que su elección de especialización estuvo influenciada por las necesidades de la comunidad, más que la propia realización personal.

En estudiantes de Irán el "altruismo y el desafío intelectual" fueron las motivaciones más influyentes en la elección de la carrera (32). En el caso de medicina ocurrió algo similar, respecto a la elección de la especialidad (23). Esta decisión estaría influenciada por muchos factores incluyendo: el desafío intelectual de la especialidad, modelos de conducta, y por supuesto: el altruismo, indistintamente entre hombres y mujeres.

\section{Carga de deuda de los estudiantes}

Un factor que influye significativamente en la elección de especializarse en los Estados Unidos, se relaciona con la carga de deuda acumulada de los estudiantes. Mientras mayor es esta carga, mayor es el tiempo que transcurre desde la graduación hasta que el profesional se matricula en algún programa de especialidad; aunque la posibilidad de especializarse se mantiene de igual manera entre los odontólogos. Sin embargo, durante los últimos años los profesionales se han visto menos influenciados por el factor económico de la deuda, debido a que se ha comprobado que la obtención de la especialidad y el ejercicio remunerado de estas, son suficientes para asumir las deudas de la educación (6).

Otro estudio (34) entre estudiantes estadounidenses realizado entre los años 2000 y 2004 concluyó que los alumnos de cuarto año que tenían: un mentor en la escuela dental, un alto promedio $\mathrm{y}$ el aliento de personas significativas; fueron más propensos a solicitar la formación de posgrado, sin importar la deuda acumulada que tuvieran. Los resultados sugieren que la tutoría y el estímulo de la facultad son influencias más importantes que la deuda, en los planes para la formación de posgrado.

Por otro lado, el $77 \%$ de estudiantes de odontología canadienses, consideró una carrera exclusivamente en odontología general debido a éste factor; pero si la deuda educativa no fuera un aspecto relevante, sólo el $12 \%$ seguiría considerando esta área, en beneficio de la especialización odontológica (33).

\section{Evolución de expectativas durante el pregrado}

Si bien la elección de la especialidad se concreta solo al terminar la carrera, la literatura describe que existen variaciones de las especialidades deseadas mientras se transita por el pregrado $(5,19,25,35,36)$ Una investigación realizada en Estados Unidos (5) entre estudiantes de diferentes universidades, concluyó que el $51 \%$ de los encuestados no planeaban especializarse, el $21 \%$ decidieron especializarse antes de entrar a la universidad, el 10\% decidió especializarse en el primer año de la escuela de odontología, el $6 \%$ lo decidió en el segundo año, el $11 \%$ lo decidió en el tercer año, y sólo el $1 \%$ lo determinó en el cuarto año.

En Estados Unidos, el 24\% de los estudiantes manifestó planes de seguir una especialidad, en comparación con el $61 \%$ que no lo hicieron y el $15 \%$ que no lo sabía (35). Mientras que otro estudio (36), también en este país, señala que entre los estudiantes de cuarto año, sólo el $65 \%$ consideró especializarse; en cambio en los niveles inferiores el porcentaje llegó hasta el $70 \%$.

Una investigación de Inglaterra sugiere que el nivel de idealismo profesional (nivel de remuneración, empleo y estabilidad) puede ser menos entre los estudiantes de último curso, respecto a los alumnos entrantes a la carrera, por lo que la obtención de una especialidad se hace tremendamente importante para alcanzar dichas metas (25). Otro estudio realizado en Brasil apoya la afirmación de la pérdida del idealismo, los estudiantes de odontología en los primeros años de sus estudios tienden a tener una visión más idílica de la profesión en comparación con los estudiantes del último año, que progresivamente durante su formación fueron construyendo una visión más mesurada acerca de la odontología (19). 
En un estudio comparativo entre estudiantes de Japón y Suecia (37), se encontró que entre los estudiantes de quinto año el $64 \%$ de japoneses y el $47 \%$ de estudiantes suecos planean trabajar como dentistas generales. Siendo el porcentaje restantes quienes si planeaban especializarse o seguir algún programa de posgrado. Al examinar a los estudiantes que deseaban especializarse, un tercio aún no había decidido una especialización específica. Además, el $37 \%$ de los estudiantes suecos estaban planeando convertirse en especialistas, probablemente porque pensaban que los especialistas ganan más dinero que los dentistas generales.

Otro estudio realizado en universidades de la India encontró que en los estudiantes de primer año se registra un menor porcentaje de quienes pretendían realizar algún estudio de posgrado, incluidas las especialidades, comparado con estudiantes de cuarto año que registran el mayor interés en realizar la especialidad; pero en el internado ocurre un fenómeno donde la intención de especializarse disminuye (17).

Aditya (8), también en India, el año 2013 evaluó la actitud de estudiantes de odontología frente a la odontología familiar o comunitaria, encontrando: que la mejor actitud frente a esta especialidad fue encontrada entre los estudiantes de cuarto año, una actitud promedio entre los de segundo y tercer año y una peor actitud entre los estudiantes de primer año.

\section{DISCUSIÓN}

En Estados Unidos durante 1970, los especialistas representaban menos del $10 \%$ de todos los dentistas en ejercicio; hoy aproximadamente el $22 \%$ de los dentistas activos son especialistas y se espera que este número aumente en las próximas décadas. Se puede decir pues, que existe una clara especialización de la profesión (33).

Referente a las razones por la que los estudiantes se especializan en la odontología, existe concordancia en que la expectativa de seguridad y un futuro profesional brillante es lo que mantiene a la mayoría de los estudiantes motivados durante el curso de su educación dental. Si a los estudiantes de odontología se les pregunta, sobre ¿qué es un futuro profesional brillante? ellos lo relacionan con seguridad en el empleo, buenos ingresos, horario de trabajo flexible, etc. (16). Aunque también existen otras motivaciones altruistas $(4,23,32)$, así como la influencia de amigos $\mathrm{y}$ familiares (38).

Sin embargo, el peso de cada uno de estos factores es variable entre los distintos países e incluso al interior de estos. En América Latina con excepción de Brasil, la investigación en esta área ha sido escasa. Esto dificulta la generación de políticas para la formación de recursos humanos especializados en odontología que satisfagan las necesidades de la población.

Por tanto, es necesario desarrollar investigación que permita comprender de mejor manera la elección de especialidad de los actuales y futuros dentistas en la realidad de cada país o región. Para esto resulta relevante conocer las especialidades que son más deseadas por los estudiantes latinoamericanos y qué factores están influyendo en esa elección, así como desarrollar intervenciones que permitan dar a conocer todas las opciones de especialización que ofrece la profesión, incluyendo aquellas de carácter prioritario para el sistema público de salud.

Debido a la creciente cantidad de estudiantes de odontología en Latinoamérica (26), la investigación en este tema puede impactar positivamente a una proporción importante de la población, considerando siempre las particularidades de cada país y sus regiones o provincias.

\section{CONCLUSIONES}

Existen diversos motivos que influencian la decisión de especializarse y la elección de la especialidad, entre ellos se cuentan: estabilidad financiera y retribución económica, realización e intereses personales, reconocimiento social o "status", experiencia profesional, altruismo y carga de deuda de los estudiantes. Sin embargo, el peso de cada uno de estos factores es variable entre los distintos países e incluso al interior de estos. En América Latina es necesario aumentar la investigación en el área para apoyar no solo el desarrollo académico, sino también el desarrollo de la salud pública odontológica. 


\section{Correspondencia:}

Ricardo Cartes-Velásquez

Roosevelt 1550, Barrio Universitario. Concepción, Chile.

Correo electrónico: cartesvelasquez@gmail.com

\section{REFERENCIAS BIBLIOGRÁFICAS}

1. Al-Dlaigan Y, AlBarakati S, Al-Habeeb F, Al-Hulaily M. Career characteristics and postgraduate education of female dentist graduates of the College of Dentistry at King Saud University, Saudi Arabia. Saudi Dent J. 2012; 24(1):29-34.

2. Lissera RG, Battellino L. Motivaciones y creencias de los estudiantes de nuevo ingreso a la carrera de odontología (Córdoba, Argentina). Rev Educ Cienc Salud. 2007; 4(2):121-8.

3. Rashid HH, Ghotane S, Abufanas S, Gallaghe J. Short and long-term career plans of final year dental students in the United Arab Emirates. BMC Oral Health 2013, 13:39.

4. Mehmood SI, Kumar A, Al-binali A, Borleffs J. Specialty preferences: trends and perceptions among saudi undergraduate medical students. Med Teach. 2012; 34(Suppl.1):51-60.

5. Dhima M, Petropoulos V, Han RK, Kinnunen T, Wright RF. Dental students perceptions of dental specialties and factors influencing specialty and career choices. J Dent Educ. 2012; 76(5):562-73.

6. Blissett R, Lee MC, Jimenez M, Sukotjo C. Differential factors that influence applicant selection of a prosthodontic residency program. J Prosthodont. 2009; 18(3):283-8.

7. Zarchy M, Kinnunen T, Chang BM, Wright R. Increasing predoctoral dental students' motivations to specialize in prosthodontics. J Dent Educ. 2011; 75(9):1236-43.

8. Aditya S. Motivations and future aspirations of dental interns: a cross-sectional study. SRM J Res Den Sci. 2013; 4(3):114-8.

9. Naidu GM, Ghanasyam PM, Kandregula CR, Suresh B, KVNR P. Choosing Public Health Dentistry as a Career: A Cross-Sectional Study. JCDR. 2014; 8(2):199-202.

10. Schenkein HA, Best AM. Factors Considered by new faculty in their decision to choose careers in academic dentistry. J Dent Educ. 2001; 65(9):832-40.

11. Aziz SR, Ziccardi VB, Chuang S. Training satisfaction versus dissatisfaction among chief residents in oral and maxillofacial surgery: a pilot survey. J Oral Maxillofac Surg. 2013; 71(5):974-80.

12. Newton P, Cabot L, Wilson HF, Gallagher JE. The graduate entry generation: a qualitative study exploring the factors influencing the career expectations and aspirations of a graduating cohort of graduate entry dental students in one London institution. BMC Oral Health. 2011; 11:25-41.

13. Newton DA, Grayson MS. Trends in career choice by US medical school graduates. JAMA. 2003; 290(9):1179-82.

14. Brotherton SE, Rockey PH, Etzel SI. US graduate medical education, 2002-2003. JAMA. 2003; 290(9):1197-202.

15. Jagsi R, Griffith KA, De Castro RA, Ubel P. Sex, role models, and specialty choices among graduates of US medical schools in 2006-2008. J Am Coll Surg. 2014; 218(3):345-52.

16. Baharvand M, Moghaddam EJ, Pouretemad H, Alavi K. Attitudes of iranian dental students toward their future careers: an exploratory study. J Dent Educ. 2011; 75(11):1489-95.

17. Del Aguila MA, Leggott PJ, Paul BR, Denise LP, Felber GD. Practice patterns among male and female general dentists in a Washington state population. J Am Dent Assoc. 2005; 136(6):790-6.

18. Garla BK. Career aspirations and reasons for choosing dentistry as a career - a survey of dental students in Gandhi Dental College and Hospital, Bhubaneswar. Ann Essences Dent. 2011; 3(2):108-10.

19. Yan $X$, Zhang $X$, Jinno $Y$, Gao J, Koyano $\mathrm{K}$ et al. Career choice and future design of dental students in China and Japan. Int Dent J. 2014; 64(2):68-75.

20. Ferraz B, Nicolau B, Muller K, Bedos C, Cilense AC. Brazilian dental students intentions and motivations towards their professional career. J Dent Educ. 2013; 77(3):337-44.

21. Saeed S, Jimenez M, Howell H, Karimbux N, Sukotjo C. Which factors influence students' selection of advanced graduate programs? one institution's experience. J Dent Educ. 2008; 72(6):689-97.

22. Ferreira N, Ferreira A, Do CM, Freire M. Mercado de trabalho na odontologia: contextualização e perspectivas job market in dentistry: historical context and perspectives. Rev Odontol UNESP. 2013; 42(4):304-9.

23. Lambert EM, Holmboe ES. The Relationship between specialty choice and gender of U.S. medical students. 1990-2003. Acad Med. 2005; 80(9):797-802.

24. Gallagher JE, Pate R1, Donaldson N, Wilson HF. The emerging dental workforce: why dentistry? A quantitative study of final year dental students' views on their professional career. BMC Oral Health. 2007; $7: 7$.

25. Gallagher J, Clarke W, Wilson N. Understanding the motivation: a qualitative study of dental students' choice of professional career. Eur J Dent Educ. 2008; 12(2):89-98. 
26. Carrizo A. Dentists' training in Chile and some iberoamerican countries. Considerations for education, health and job. J Oral Res. 2014; 3(2):119-22.

27. Tanalp J, Ilguy D, Dikbas D, Oktay I. Demographic profile and future expectations of students enrolled in a Turkish private dental school. J Dent Educ. 2012; 76(6):800-9.

28. Aggarwal A, Mehta S, Gupta D, Sheikh S, Pallagatti S, Singh R et al. Dental students motivations and perceptions of dental professional career in India. J Dent Educ. 2012; 76(11):1532-9.

29. Gambetta K, Mariño R, Morgan M. Socio-demographic characteristics and career choices amongst Chilean dental students. J Oral Res 2014; 3(2):83-9.

30. Fuoad SA. a trend of post graduate clinical training in general dentistry: a review. RRJDS. 2014; 2 (1):57-62.

31. Dastjerdi MV, Mahdian M, Dastjerdi EV, Namdari M. Study motives and career choices of Iranian medical and dental students. Acta Med Iran. 2012; 50(6):41724.

32. Khami MR, Murtomaa H, Jafarian M, Vehkalahti MM, Virtanen JI. Study motives and career choices of Iranian dental students. Med Princ Pract. 2008;17(3): 221-6.

33. Nashleanas BM, McKernan SC, Kuthy RA, Qian F. Career influences among final year dental students who plan to enter private practice. BMC Oral Health, 2014; 14:18.
34. Scarbecz M, Ross JA. The relationship between gender and postgraduate aspirations among firstand fourth-year students at public dental schools: a longitudinal analysis. J Dent Educ. 2007; 71(6):797809.

35. Rupp JK, Jones DL, Seale NS. Dental students' knowledge about careers in academic dentistry. J Dent Educ. 2006; 70(10):1051-60.

36. Walton JN, Pros C, Matthew IR, Dumaresq C, Sudmant W. The burden of debt for Canadian dental students: Part 4. The influence of debt on program and career decisions. J Can Dent Assoc. 2006; 72(10):913.

37. Karibe H, Kawakami T, Suguki A, Warita S, Ogata K, Aoyagi $\mathrm{K}$ et al. Career choice and attitudes towards dental education amongst dental students in Japan and Sweden. Eur J Dent Educ. 2009; 13(2):80-6.

38. Halawany H. Career motivations, perceptions of the future of dentistry and preferred dental specialties among saudi dental students. Open Dent J. 2014; 8:12935 .

Recibido: 18/12/2014

Aceptado: 20/02/2015 